\begin{tabular}{|c|l|}
\hline Title & A model evaluating effect of disaster warning issuance conditions on "cry wolf syndrome" in the case of a landslide \\
\hline Author(s) & Uchida, Kenetsu \\
\hline Citation & $\begin{array}{l}\text { European Journal of Operational Research, 218(2), 530-537 } \\
\text { https://doi.org/40.1016/.ejor.2011.10.050 }\end{array}$ \\
\hline Issue Date & 2012-04_16 \\
\hline Doc URL & http://hdl.handle.net/2115/49005 \\
\hline Type & article (author version) \\
\hline File Information & EJOR2182_530-537.pdf \\
\hline
\end{tabular}

Instructions for use 


\title{
A model evaluating effect of disaster warning issuance conditions on "cry wolf syndrome" in the case of a landslide
}

\author{
Kenetsu Uchida, Hokkaido University Graduate School of Engineering \\ Corresponding author: Kenetsu Uchida, E-mail address: uchida@eng.hokudai.ac.jp \\ Address: North 13 West 8 Kita-ku Sapporo, Japan, 060-8628. \\ Tel. +81-11-706-6214; fax: +81-11-706-6214.
}

\begin{abstract}
This study proposes a model that clarifies how disaster warning issuance conditions affect "cry wolf" syndrome. The disaster assumed in this study is landslide caused by heavy rainfall. Local authorities that issue disaster warnings are thought to tend to avoid the situation where casualty occurs without the issuance to residents of a disaster warning. As a result, the issuance conditions may be relaxed. Under this circumstance, however, the residents are thought to tend to ignore disaster warnings, since such warnings are inaccurate. Thus may emerge the "cry wolf" syndrome. In this study, a simulation model that expresses the behaviors of the local authority and the residents has been developed. For the purpose of demonstrating the model, numerical experiments were then carried out. In the numerical experiments, the effects of optimal issuance conditions for disaster warnings on the cost incurred by the resident were evaluated by using assumed parameters for the model.
\end{abstract}

Keywords: (I) Decision support system, (D) OR in societal problem analysis, "cry wolf" syndrome, disaster warning issuance conditions

\section{INTRODUCTION}

Disaster warnings are regarded as an important measure for mitigating the casualty caused by natural disasters. The local authority tends to be afraid of the situation where a natural disaster occurs when a disaster warning has not been issued to residents. Therefore, the local authority may find itself in the dilemma of whether or not to relax the disaster warning issuance conditions. If the local authority relaxes those conditions, the residents will tend to discount the warnings, since the warning will then be a less accurate predictor of natural disaster. Thus, it is thought that "cry wolf" syndrome can occur. In "cry wolf" syndrome, residents who at first evacuate when disaster warnings are issued tend to become less likely to evacuate after over-frequent false alarms, since the cumulative costs required for evacuation, i.e. physical 
cost and psychological cost, can be perceived as greater under overly frequent false alarms than that under adequately frequent alarms.

Studies on "cry wolf" syndrome in emergencies have focused on various aspects of the syndrome. Nakatani et al. (2009a,b) experimentally analyzed the effects of various sound parameters for auditory warning signals on human behavior in an emergency for the purpose of preventing "cry wolf" syndrome. Wolshon et al. (2005) reviewed the transportation engineering aspects of hurricane evacuations. They noted that excessive evacuation calls have the potential to create "cry wolf" syndrome, in which people who are ordered to evacuate too often when hazardous conditions do not actually materialize are less likely to evacuate in the future. Atwood and Major (1998) investigated the effect of false alarms on "cry wolf" syndrome. These two studies showed that an appropriate evacuation order requires a balance between the level of risk and potential consequences so as not to adversely affect future evacuation orders. Dash and Gladwin (2007) reviewed the literature on a wide range of factors that affect evacuation decisions after people hear hurricane forecasts and other information. They focused on three broad areas of research that often overlap: warning, risk perception and evacuation research. They mentioned that "cry wolf" is an evacuation order for a storm that misses. Sorensen (2000) mentioned that the likelihood of people responding to a warning is not diminished by what has come to be labeled the "cry-wolf", syndrome if the basis of the false alarm is understood, although repetitive false alarms may decrease response.

Tamura et al. (2000) showed that a value function under risk is useful for modeling low-probability, high-consequence events like earthquakes for which expected utility theory is inadequate. They also showed that using the value function under risk is an appropriate approach for modeling and analyzing decision-making regarding low-probability, high-consequence events. Yi and Özdamar (2007) proposed an integrated location-distribution model for coordinating logistics support and evacuation operations in disaster response activities. The proposed model considered both the selection of sites that result in maximum coverage of medical need in affected areas and the medical personnel who are on duty in nearby hospitals have to be re-shuffled to serve both temporary and permanent emergency units. Lindell (2008) described a simple, rapid method for calculating evacuation time estimates (ETEs) that was compatible with research findings about evacuees' behavior in hurricanes. Saadatseresht et al. (2009) proposed a three-step approach for evacuation planning. In their study, both multiobjective evolutionary algorithms (MOEA) and the geographical information system (GIS) were used for obtaining evacuation planning. Kailiponi (2010) used multi-attribute utility theory to propose a preliminary decision model for evacuation decision. The effect of differences in forecast precision on the optimal evacuation decision was investigated in the study. Bretschneider and Kimms (2011) presented a two-stage heuristic solution approach for a pattern-based mixed integer dynamic network flow model by which traffic routing such 
that the evacuees leave the evacuation area as safe as possible and as early as possible within the considered time horizon was obtained. Lindell et al. (2011) extended previous research associated with households' hurricane evacuation decision making by reporting data on other aspects of evacuation logistics such as departure timing, vehicle use, evacuation routes, travel distance, shelter type, evacuation duration, and evacuation cost.

In this study, a simulation model which expresses the relationship between the local authority who issues disaster warnings and the residents who decide whether to evacuate based on those warnings is proposed. The model reproduces "cry wolf" syndrome, since it factors in the learning process of the resident. For the purpose of demonstrating the model, numerical experiments will be then carried out. In the numerical experiments, the effects of optimal issuance conditions for disaster warnings on the cost incurred by the resident will be calculated by using assumed parameters which are associated with the model. The optimal issuance conditions are determined so as to maximize the expected utility (satisfaction) of the resident.

\section{MODEL FORMULATION}

The natural disaster addressed in the present study is landslide caused by heavy rainfall. We set the following four definitions regarding the disaster warnings issued to the residents.

We consider only a landslide that occurs on days when the daily rainfall intensity (DRI) $q$ is:

$$
q_{1} \leq q \leq q_{\max } .
$$

An alert $\left(r_{1}\right)$ is issued to residents when DRI reaches $q_{1}$.

An evacuation advisory $\left(r_{2}\right)$ is issued to residents when DRI reaches $q_{2}\left(>q_{1}\right)$.

An evacuation order $\left(r_{3}\right)$ is issued to residents when DRI reaches $q_{3}\left(q_{2}<q_{3} \leq q_{\max }\right)$.

We first define the variable $f$, which expresses the situation of DRI:

$$
f=\left\{\begin{array}{ll}
1 & \text { if } q \geq q_{1} \\
0 & \text { otherwise }
\end{array} .\right.
$$

Let $p_{f}$, where $f=1$, denote the probability of DRI being larger than $q_{1}$, which is given by:

$$
p_{f}=\operatorname{Pr}\left(q \geq q_{1}\right) \text {. }
$$

Here we address only the days when DRI is greater than or equal to $q_{1}$. Therefore, the variable $f$ which is also put beside a probability as a subscript takes $1 . p_{r_{\mathrm{i}} \mid f}$ is given by:

$$
p_{r_{1} \mid f}=\operatorname{Pr}\left(q_{1} \leq q<q_{2}\right),
$$

Let $p_{r_{\mathrm{I}} \mid f}$ denote the probability of a day when the alert is issued to the residents. In the same manner, we define the following two probabilities:

$$
\begin{aligned}
& p_{r_{2} \mid f}=\operatorname{Pr}\left(q_{2} \leq q<q_{3}\right), \\
& p_{r_{3} \mid f}=\operatorname{Pr}\left(q_{3} \leq q<q_{\max }\right) .
\end{aligned}
$$

The probabilities shown by Eqs. (2)-(4) take the values more than 0 if $f=1$ and 0 otherwise. On any 
given day, according to the probabilities shown by Eqs. (3) and (4), the evacuation advisory and the evacuation order are issued, respectively. $q_{\max }$, which is used in the assumptions, is a constant such that:

$$
p_{q_{\text {max }} \mid f}=\varepsilon,
$$

where

$$
p_{q_{\max } \mid f}=\operatorname{Pr} .\left(q \geq q_{\max }\right),
$$

and $\varepsilon>0$ is a sufficiently small constant for representing 0 that is introduced to avoid calculating infinity when we assume a theoretical probability function, e.g. $q_{\max \mid f}=\infty$ under $p_{q_{\max } \mid f}=0$. We assume that $q_{1}$, which is also used in the assumptions, is a constant that can be determined empirically. Note that $p_{q_{1} \mid f}=1$ conditional on $f=1 . \quad p_{r_{i} \mid f}(i=1, \ldots, 3)$ are then (see also Fig. 1):

$$
p_{r_{i} \mid f}=\left\{\begin{array}{cc}
p_{q_{i} \mid f}-p_{q_{i+1} \mid f} & \text { if } i=1,2 \\
p_{q_{i} \mid f} & \text { if } i=3
\end{array} .\right.
$$

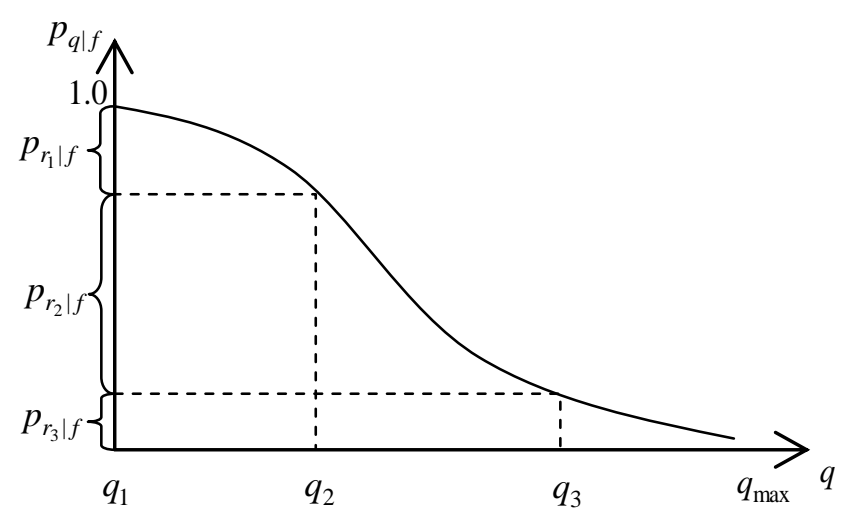

Fig. 1. Rainfall distribution

Note that since the curve shown in Fig. 1 is the cumulative density function of rainfall distribution conditional on $f=1$, the length of line segments makes sense rather than area under the curve. We assume then that landslide magnitude is conditioned on rainfall. Let $p_{m_{j} r_{i}}$ denote the probability of the landslide with the casualty level of $m_{j}(j=0, \ldots, 2)$ occurring conditional on the issuance of $r_{i}(i=1, \ldots, 3)$. Level of $m_{0}$ means no casualty. By using the probabilities of landslides with the casualties of $m_{j}(j=0, \ldots, 2)$ occurring conditional on $f=1\left(p_{m_{j} \mid f}(q)\right.$ which are function of $\left.q\right), p_{\left.m_{j}\right|_{i}}\left(\forall m_{j}, \forall r_{i}\right)$ are given by the following (see also Fig. 2):

$$
p_{m_{j} \mid r_{i}}=\left\{\begin{array}{cc}
\int_{q_{j}}^{q_{j+1}} p_{m_{j} \mid f}(x) d x /\left(q_{j+1}-q_{j}\right) & \text { if } j=0 \\
\int_{q_{j}}^{q_{j+1}}\left(p_{m_{j} \mid f}(x)-p_{m_{j-1} \mid f}(x)\right) d x /\left(q_{j+1}-q_{j}\right) & \text { if } j=1 . \\
\int_{q_{j}}^{q_{\max }}\left(1-p_{m_{j-1} \mid f}(x)\right) d x /\left(q_{\max }-q_{j}\right) & \text { if } j=2
\end{array}\right.
$$

We assume that both $p_{q_{i} \mid f}$ and $p_{m_{j} \mid f}(q)$ can be estimated from historical data. 


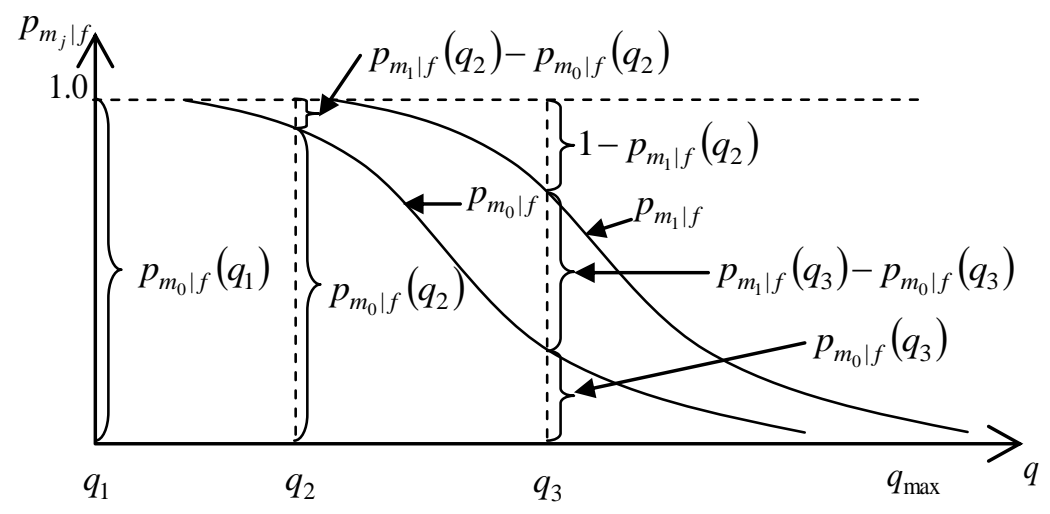

Fig. 2. Probability of landslide

Next, we consider the costs related to the occurrence of the landslide that the resident has to pay. In this study, we address the cost required for evacuating and the cost of landside casualty in the event of not evacuating. We do not address the cost of property damage resulting from landslide. Let $l_{m_{j}}$ denote the expected cost for personal suffering incurred by the resident who does not evacuate conditional on the occurrence of $m_{j}$. In contrast, the resident who evacuates at this time does not have to pay for personal suffering; instead, the resident has to pay the expected cost $\left(l_{e}\right)$ required for evacuation, which is the psychological cost plus the physical cost. It is assumed that the residents decide whether to evacuate after receiving the disaster warning $r_{i}$ issued by the local authority. Let $p_{e \mid r_{i}}(d)$ denote the probability of the resident evacuating conditional on the issuance of $r_{i}$ on day $d . p_{e \mid r_{i}}(d=0)$ is then the initial probability of the resident evacuating conditional on the issuance of the disaster warning $r_{i}$. The initial probability of the resident not evacuating is then $p_{s \mid r_{i}}(0)=1-p_{e \mid r_{i}}(0)$. The residents are assumed to make evaluations of the decisions in retrospect, i.e. evaluations after the fact. Then, the residents assumed in the present study change their evacuation probability on the next day $p_{e \mid r_{i}}(d+1)$ based on the evaluations they made. Their decisions are evaluated as correct if a landslide with casualty greater than or equal to $m_{1}$ occurs when they evacuate, and if a landslide with casualty greater than or equal to $m_{1}$ does not occur when they do not evacuate. Otherwise, their decisions are evaluated as incorrect. By using $m_{j}$ which is given after the fact, the residents are assumed to change their evacuation probabilities on the next day conditional on the issuance of $r_{i}$ as shown by:

$$
p_{e \mid r_{i}}(d+1)=\left\{\begin{array}{cc}
\alpha \cdot p_{e \mid r_{i}}(d)+(1-\alpha) & \text { if } m_{j}(j=1,2) \\
\alpha \cdot p_{e \mid r_{i}}(d) & \text { otherwise }
\end{array}\right.
$$

where $\alpha(0 \leq \alpha \leq 1)$ is a parameter of the learning process. The smaller the parameter is, the stronger the learning effect is. Note that no change is made to the probability of the resident evacuating when no disaster warning is issued. It is evident that the resident learns not only from experiences with the events but also from other residents. Since this study focuses on the learning process based on experience, the latter effect which is although important in real situation has not been introduced to the formulation. 
Based on the formulation shown above, the expected cost that the resident has to pay on day $d$ is:

$$
E[g(d)]=\left\{\begin{array}{cc}
\sum_{j} \sum_{i} p_{f} \cdot p_{r_{i} \mid f} \cdot p_{m_{j} \mid r_{i}} \cdot\left(p_{e \mid r_{i}}(d) \cdot l_{e}+p_{s \mid r_{i}}(d) \cdot l_{m_{j}}\right) & \text { if } f=1 \\
0 & \text { otherwise }
\end{array} .\right.
$$

The expected cost that the resident has to pay during year $t$ is given by:

$$
E[g(t)]=\sum_{d=365(t-1)}^{365 t} E[g(d)] .
$$

The present value of the expected cost that the resident has to pay during $T$ years is:

$$
E[G(T)]=\sum_{t=1}^{T} \frac{E[g(t)]}{(1+r)^{t-1}}
$$

where $r$ is discount rate.

The present value of the expected cost paid by the resident $G(T)$ in Eq. (12) is difficult to calculate analytically since the evacuation probability $\left(p_{e \mid r_{i}}(d)\right)$ can vary every day depending on the evaluations of the decisions the resident makes. For the purpose of analyzing "cry wolf" syndrome, the introduction of the dynamics of $p_{e \mid r_{i}}(d)$ is necessary. The estimation of $G(T)$ under the dynamic process of $p_{e \mid r_{i}}(d)$ is important to determine the disaster warning issuance conditions. This study applies a Monte Carlo simulation technique to calculate the resident's expected cost $G(T)$. It is easily imagined that if the issuance conditions are relaxed (e.g., issuing $r_{i}$ at $q_{i}-\delta q_{i}\left(\delta q_{i}>0, i=1,2,3\right)$ ), the residents might ignore the disaster warnings since their accuracy would be lower. If a landslide with greater casualty than $m_{0}$ occurs under such circumstances, the costs paid by the residents can be huge, since most of the residents are considered as not evacuating. In contrast, it is easily imagined that if the issuance conditions are tightened (e.g., issuing $r_{i}$ at $q_{i}+\delta q_{i}\left(\delta q_{i}>0, i=1,2,3\right)$ ), the costs for the residents would be also be huge since there is the relative large possibility of a landslide with greater casualty than $m_{0}$ occurring before disaster warnings are issued. Therefore, it is useful to calculate the optimal conditions of disaster warning issuance. The next section addresses the optimal issuance conditions for the residents.

\section{CONDTIONS OF DISASTER WARNING ISSUANCE}

As explained in the previous section, the residents decide their behaviors (evacuate or not) and change their evacuation probabilities based on experience of disaster warning issuance. This section investigates the optimal conditions for disaster warning issuance. Since the DRI of $q_{1}$, which is the issuance conditions of the alert, $r_{1}$, is predefined, the local authority is assumed to determine the DRIs of $q_{2}$ and $q_{3}$ by which the evacuation advisory $\left(r_{2}\right)$ and the evacuation order $\left(r_{3}\right)$ are respectively issued. The local authority is assumed to determine $q_{2}$ and $q_{3}$ such as to minimize the inclusive cost paid by the resident. The local authority is assumed to not be able to know the evacuation probability $p_{e \mid r_{i}}$ when the 
disaster warning $r_{i}$ is issued. Therefore, the local authority has to estimate the evacuation probability first. The discussions in this section will proceed by assuming that the local authority has determined the vector of DRIs $\mathbf{q}=\left(\begin{array}{ll}q_{2} & q_{3}\end{array}\right)^{T}$. At the point in time when the observed DRI reaches $q_{i}$, the corresponding disaster warning $r_{i}$ is automatically issued. The estimation of the probability of a landslide with casualty $m_{j}$ occurring $\left(p_{m_{j} \mid r_{i}}\right)$ for a given $\mathbf{q}=\left(\begin{array}{ll}q_{2} & q_{3}\end{array}\right)^{T}$ is thought to be possible by using historical data. For any $i \in\{1,2,3\}$, we denote the probabilities as:

$$
p_{m_{j} \mid r_{i}}=p_{m_{j} \mid r_{i}}\left(q_{i}, q_{i+1}\right)
$$

where

$$
q_{4}=q_{\max } .
$$

The local authority is assumed to estimate two utility functions of the resident: the utility perceived when the resident evacuates and the utility perceived when the resident does not evacuate, under the issuance of $r_{i}$. Let us consider first the situation where a landslide occurs under the issuance of disaster warning $r_{i}$. In this case, the resident who does evacuate has to pay the cost required for evacuating. The resident who does not evacuate has to pay the cost of landslide casualty. Consider next the situation where a landslide does not occur under the issuance of disaster warning $r_{i}$. In this case, the resident who evacuates has to pay the cost required for evacuating. On the other hand, the resident who does not evacuate has to pay no cost. Table 1 shows the relationship between the behaviors the resident takes and the gains the resident receives. By using the relationship shown in Table 1, the utility functions under the issuance of disaster warning $r_{i}$ when the resident evacuates or does not are respectively given by:

$$
\begin{aligned}
& u_{e \mid r_{i}}\left(q_{i}, q_{i+1}\right)=-l_{e}+a_{e}, \\
& u_{s \mid r_{i}}\left(q_{i}, q_{i+1}\right)=-\sum_{j=1}^{2} p_{m_{j} \mid r_{i}}\left(q_{i}, q_{i+1}\right) \cdot l_{m_{j}},
\end{aligned}
$$

where $a_{e}$ is the alternative specific constant of the utility perceived when evacuating which is to be formulated later.

Table 1. Utility under the issuance of a disaster warning

\begin{tabular}{|c|c|c|c|}
\hline \multirow{2}{*}{$\begin{array}{c}\text { disaster warning } \\
\text { issued }\end{array}$} & $\begin{array}{c}\text { probability of casualty level } \\
m_{j} \text { under the issuance of } r_{i}\end{array}$ & evacuation & expected gain \\
\hline \multirow{3}{*}{$r_{i}$} & $p_{m_{0} \mid r_{i}}\left(q_{i}, q_{i+1}\right)$ & Yes & $-p_{m_{0} \mid r_{i}}\left(q_{i}, q_{i+1}\right) \cdot l_{e}$ \\
\cline { 2 - 4 } & $p_{m_{1} \mid r_{i}}\left(q_{i}, q_{i+1}\right)$ & No & 0 \\
\cline { 2 - 4 } & $p_{m_{2} \mid r_{i}}\left(q_{i}, q_{i+1}\right)$ & Yes & $-p_{m_{1} \mid r_{i}}\left(q_{i}, q_{i+1}\right) \cdot l_{e}$ \\
\cline { 2 - 4 } & & No & $-p_{m_{1} \mid r_{i}}\left(q_{i}, q_{i+1}\right) \cdot l_{m_{1}}$ \\
\cline { 2 - 4 } & & Yes & $-p_{m_{2} \mid r_{i}}\left(q_{i}, q_{i+1}\right) \cdot l_{e}$ \\
\cline { 2 - 4 } & & & $-p_{m_{2} \mid r_{i}}\left(q_{i}, q_{i+1}\right) \cdot l_{m_{2}}$ \\
\hline
\end{tabular}


According to random utility theory (e.g., Daganzo 1979), if the independently and identically distributed Gumbel distributions are applied to the random terms for two utility functions, the evacuating probability are then:

$$
p_{e \mid r_{i}}\left(q_{i}, q_{i+1}\right)=\frac{1}{1+\exp \left(\theta \cdot\left(u_{s \mid r_{i}}\left(q_{i}, q_{i+1}\right)-u_{e \mid r_{i}}\left(q_{i}, q_{i+1}\right)\right)\right)}, p_{s \mid r_{i}}\left(q_{i}, q_{i+1}\right)=1-p_{e \mid r_{i}}\left(q_{i}, q_{i+1}\right)
$$

where $\theta$ is a dispersion parameter. The evacuating probabilities shown by Eq. (17) can be obtained by assuming the resident's expected utility maximization behavior. If the alternative specific constant, $a_{e}$, is not introduced to the utility function, the probability of the resident evacuating shown by Eq. (17) can be very small compared with that observed in real situation since the probability of casualty occurring are in general very small in the case of landslide. Therefore, we introduced the alternative specific constant. The alternative specific constant can be determined by both the disaster warning issuance conditions and the cost of landslide casualty. The alternative specific constant assumed in this study is calculated based on an assumption, which is originally made in this study, that the resident who does evacuate additionally receives the benefit which is proportional to the costs associated with not evacuating (i.e., the cost of landslide casualty) since the resident can avoid paying that. Thus, the alternative specific constant is given by:

$$
a_{e}=\beta \cdot \sum_{j=1}^{2} p_{m_{j} r_{i}}\left(q_{i}, q_{i+1}\right) \cdot l_{m_{j}},
$$

where $\beta$ is a proportional constant which can be estimated by using SP data. For simplicity, we assume $\beta=1$ in the rest of the paper. For a given disaster warning issuance condition, $q_{i}(\forall i \in\{1,2,3,4\})$, the resident maximizes the expected utilities which are calculated by using the sets of $\left(q_{i}, q_{i+1}\right)(\forall i \in\{1,2,3\})$. The expected maximum utilities which are given by:

$$
S_{r_{i}}\left(q_{i}, q_{i+1}\right)=\frac{1}{\theta} \cdot \ln \left(\sum_{c \in\{e, s\}} \exp \left(u_{c \mid r_{i}}\left(q_{i}, q_{i+1}\right)\right)\right)(\forall i \in\{1,2,3\}),
$$

accompanied by the occurrence probabilities of $p_{r_{i} \mid f}(\mathbf{q})(\forall i \in\{1,2,3\})$. The local authority is assumed to determine $\mathbf{q}=\left(\begin{array}{ll}q_{2} & q_{3}\end{array}\right)^{T}$, such as to maximize the expected utility. The local authority's determination is equivalent to minimizing the inclusive cost paid by the resident. The problem for the local authority becomes:

$$
\begin{aligned}
& \max S(\mathbf{q})=\sum_{i=1}^{3} p_{r_{i} \mid f}(\mathbf{q}) \cdot S_{r_{i}}\left(q_{i}, q_{i+1}\right), \\
& \text { s.t. } \quad\left(q_{1} \leq\right) q_{2}<q_{3}\left(\leq q_{\max }\right) .
\end{aligned}
$$

Under the issuance of $r_{i}$, resident's utilities are expressed by random variables. Each resident compares the utility of evacuation to the utility of staying and makes an evacuation decision on that basis. The resident will decide whether to evacuate based on the evacuation probability. The expected value of the maximum utility under the issuance of $r_{i}$,which is denoted by Eq. (19), expresses the satisfaction perceived by the resident. Note that the problem may not always minimize the actual cost of the landslide incurred by the resident, since we address the perceived cost. Since it is obvious from Fig. 1 that $p_{r_{i} \mid f}$ is 
a function of $\mathbf{q}=\left(\begin{array}{ll}q_{2} & q_{3}\end{array}\right)^{T}$, then $p_{r_{i} \mid f}$ is replaced by $p_{r_{i} \mid y}(\mathbf{q})$ in Eq. (19). As explained in the earlier section, the local authority tends to avoid the situation of landslide casualties in the absence of disaster warning issuance. The local authority can avoid such situation by relaxing the conditions for disaster warning issuance, but then the residents may ignore the disaster warnings since warnings under such issuance conditions are inaccurate. If the landslide with large casualty occurs under such a situation, the costs to the residents can be huge. The problem formulated above does not provide such solutions. The reasons are as follows. It is obvious from the formulation that $u_{e \mid r_{i}}\left(q_{i}, q_{i+1}\right)$ and $u_{s \mid r_{i}}\left(q_{i}, q_{i+1}\right)$ are the increasing function and the decreasing function with respect to $q_{i}$ and $q_{i+1}$, respectively. Therefore, $S_{r_{i}}\left(q_{i}, q_{i+1}\right)$ is the increasing function of both $q_{i}$ and $q_{i+1}$. Therefore, if the local authority tightens the issuance conditions for disaster warnings, the satisfaction perceived by the resident under the issuance of $r_{i}$, i.e. $S_{r_{i}}\left(q_{i}, q_{i+1}\right)$, is greater at the beginning. However, since $p_{r_{i} \mid f}(\mathbf{q})$ changes simultaneously corresponding to the issuance conditions $\mathbf{q}=\left(\begin{array}{ll}q_{2} & q_{3}\end{array}\right)^{T}$, then the excessive tightening of $\mathbf{q}=\left(\begin{array}{ll}q_{2} & q_{3}\end{array}\right)^{T}$ does not provide us the maximum of $S(\mathbf{q})$. Thus, the problem denoted by Eqs. (20) and (21) can provide us an interior solution, i.e. $q_{1} \neq q_{2}<q_{3} \neq q_{\max }$.

Next we consider the uniqueness of solution. From Fig. 1, which shows $p_{r_{i} \mid f}(i=1, \ldots, 3)$, we obtain:

$$
\begin{aligned}
& a \cong \frac{\partial p_{r_{1} \mid f}\left(q_{2}\right)}{\partial q_{2}}=-\frac{\partial p_{r_{2} \mid f}\left(q_{2}, q_{3}\right)}{\partial q_{2}}(>0), \\
& b \cong \frac{\partial p_{r_{3} \mid f}\left(q_{3}\right)}{\partial q_{3}}=-\frac{\partial p_{r_{2} \mid f}\left(q_{2}, q_{3}\right)}{\partial q_{3}}(<0) .
\end{aligned}
$$

By using the above relationships, and by performing a first-order expansion to $p_{r_{i} \mid y}$ at $\mathbf{q}^{*}=\left(\begin{array}{ll}q_{2}^{*} & q_{3}^{*}\end{array}\right)$, we obtain:

$$
\begin{aligned}
p_{r_{1} \mid f}\left(q_{2}^{*}+\Delta q_{2}\right) & \approx p_{r_{1} \mid f}\left(q_{2}^{*}\right)+\left.\frac{\partial p_{r_{1} \mid f}\left(q_{2}\right)}{\partial q_{2}}\right|_{\mathbf{q}=\mathbf{q}^{*}} \cdot \Delta q_{2} \\
& =p_{r_{1} \mid f}\left(q_{2}^{*}\right)+\left.a\right|_{\mathbf{q}=\mathbf{q}^{*}} \cdot \Delta q_{2} \\
p_{r_{2} \mid f}\left(q_{2}^{*}+\Delta q_{2}, q_{3}^{*}+\Delta q_{3}\right) & \approx p_{r_{2} \mid f}\left(q_{2}^{*}, q_{3}^{*}\right)-\left.\frac{\partial p_{r_{1} \mid f}\left(q_{2}\right)}{\partial q_{2}}\right|_{\mathbf{q}=\mathbf{q}^{*}} \cdot \Delta q_{2}-\left.\frac{\partial p_{r_{3} \mid f}\left(q_{3}\right)}{\partial q_{3}}\right|_{\mathbf{q}=\mathbf{q}^{*}} \cdot \Delta q_{3}, \\
& =p_{r_{2} \mid f}\left(q_{2}^{*}, q_{3}^{*}\right)-\left.a\right|_{\mathbf{q}=\mathbf{q}^{*}} \cdot \Delta q_{2}-\left.b\right|_{\mathbf{q}=\mathbf{q}^{*}} \cdot \Delta q_{3} \\
p_{r_{3} \mid f}\left(q_{3}^{*}+\Delta q_{3}\right) & \approx p_{r_{1} \mid f}\left(q_{3}^{*}\right)+\left.\frac{\partial p_{r_{3} \mid f}\left(q_{3}\right)}{\partial q_{3}}\right|_{\mathbf{q}=\mathbf{q}^{*}} \cdot \Delta q_{3} \\
& =p_{r_{1} \mid f}\left(q_{3}^{*}\right)+\left.b\right|_{\mathbf{q}=\mathbf{q}^{*}} \cdot \Delta q_{3},
\end{aligned}
$$

where we do not regard both $q_{1}$ and $q_{4}$ as variables, since they are the predefined constants. By 


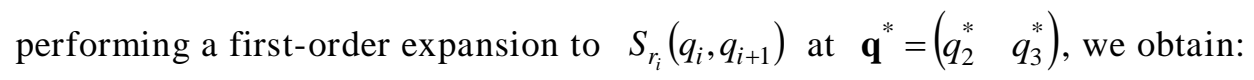

$$
\begin{aligned}
& S_{r_{1}}\left(q_{2}^{*}+\Delta q_{2}\right) \approx S_{r_{1}}\left(q_{2}^{*}\right)+\left.d_{1}\right|_{\mathbf{q}=\mathbf{q}^{*}} \cdot \Delta q_{2}, \\
& S_{r_{2}}\left(q_{2}^{*}+\Delta q_{2}, q_{3}^{*}+\Delta q_{3}\right) \approx S_{r_{2}}\left(q_{2}^{*}, q_{3}^{*}\right)+\left.c_{2}^{2}\right|_{\mathbf{q}=\mathbf{q}^{*}} \cdot \Delta q_{2}+\left.c_{2}^{3}\right|_{\mathbf{q}=\mathbf{q}^{*}} \cdot \Delta q_{3}, \\
& S_{r_{3}}\left(q_{3}^{*}+\Delta q_{3}\right) \approx S_{r_{3}}\left(q_{3}^{*}\right)+\left.d_{3}\right|_{\mathbf{q}=\mathbf{q}^{*}} \cdot \Delta q_{3},
\end{aligned}
$$

where

$$
c_{2}^{2}=\frac{\partial S_{r_{2}}\left(q_{2}, q_{3}\right)}{\partial q_{2}}, c_{2}^{3}=\frac{\partial S_{r_{2}}\left(q_{2}, q_{3}\right)}{\partial q_{3}}, d_{1}=\frac{\partial S_{r_{1}}\left(q_{2}\right)}{\partial q_{2}}, d_{3}=\frac{\partial S_{r_{3}}\left(q_{3}\right)}{\partial q_{3}} .
$$

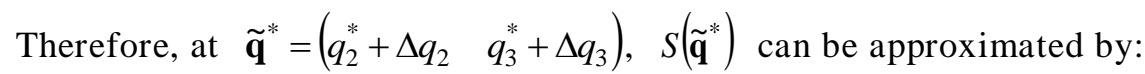

$$
\begin{aligned}
S\left(\tilde{\mathbf{q}}^{*}\right) \approx & p_{r_{1} \mid f}\left(q_{2}^{*}+\Delta q_{2}\right) \cdot S_{r_{1}}\left(q_{2}^{*}+\Delta q_{2}\right) \\
& +p_{r_{2} \mid f}\left(q_{2}^{*}+\Delta q_{2}, q_{3}^{*}+\Delta q_{3}\right) \cdot S_{r_{2}}\left(q_{2}^{*}+\Delta q_{2}, q_{3}^{*}+\Delta q_{3}\right) \\
& +p_{r_{3} \mid f}\left(q_{3}^{*}+\Delta q_{3}\right) \cdot S_{r_{3}}\left(q_{3}^{*}+\Delta q_{3}\right) \\
& =\left(p_{r_{1} \mid f}\left(q_{2}^{*}\right)+a \cdot \Delta q_{2}\right) \cdot\left(S_{r_{1}}\left(q_{2}^{*}\right)+d_{1} \cdot \Delta q_{2}\right) \\
& +\left(p_{r_{2} \mid f}\left(q_{2}^{*}, q_{3}^{*}\right)-a \cdot \Delta q_{2}-b \cdot \Delta q_{3}\right) \cdot\left(S_{r_{2}}\left(q_{2}^{*}, q_{3}^{*}\right)+c_{2}^{2} \cdot \Delta q_{2}+c_{2}^{3} \cdot \Delta q_{3}\right) \\
& +\left(p_{r_{3} \mid f}\left(q_{3}^{*}\right)+b \cdot \Delta q_{3}\right) \cdot\left(S_{r_{3}}\left(q_{3}^{*}\right)+d_{3} \cdot \Delta q_{3}\right) \\
& =A \cdot\left(\Delta q_{2}\right)^{2}+B \cdot\left(\Delta q_{3}\right)^{2}+C \cdot \Delta q_{2} \cdot \Delta q_{3}+D \cdot \Delta q_{2}+E \cdot \Delta q_{3}+F,
\end{aligned}
$$

where

$$
A=a \cdot\left(d_{1}-c_{2}^{2}\right), B=b \cdot\left(d_{3}-c_{2}^{3}\right),
$$

and $C \sim F$ are constants. According to random utility theory, we obtain:

$$
\frac{\partial S_{r_{i}}}{\partial u_{c \mid r_{i}}}=p_{c r_{i}} \forall c \in\{e, s\}
$$

$d_{1}$ and $c_{2}^{2}$ are then respectively given by:

$$
\begin{aligned}
d_{1} & =\frac{\partial S_{r_{1}}\left(q_{2}\right)}{\partial u_{e \mid r_{1}}} \cdot \frac{\partial u_{e r_{1}}}{\partial q_{2}}+\frac{\partial S_{r_{1}}\left(q_{2}\right)}{\partial u_{s \mid r_{1}}} \cdot \frac{\partial u_{s \mid r_{1}}}{\partial q_{2}} \\
& =p_{e \mid r_{1}} \cdot \frac{\partial u_{e \mid r_{1}}}{\partial q_{2}}-p_{s \mid r_{1}} \cdot \frac{\partial u_{e \mid r_{1}}}{\partial q_{2}} \\
& =\left(p_{e \mid r_{1}}-p_{s \mid r_{1}}\right) \cdot \frac{\partial u_{e \mid r_{1}}}{\partial q_{2}}, \\
c_{2}^{2} & =\frac{\partial S_{r_{2}}\left(q_{2}, q_{3}\right)}{\partial u_{e \mid r_{2}}} \cdot \frac{\partial u_{e \mid r_{2}}}{\partial q_{2}}+\frac{\partial S_{r_{2}}\left(q_{2}, q_{3}\right)}{\partial u_{s \mid r_{2}}} \cdot \frac{\partial u_{s r_{2}}}{\partial q_{2}} \\
& =p_{e \mid r_{2}} \cdot \frac{\partial u_{e \mid r_{2}}}{\partial q_{2}}-p_{s \mid r_{2}} \cdot \frac{\partial u_{e \mid r_{2}}}{\partial q_{2}} \\
& =\left(p_{e \mid r_{2}}-p_{s \mid r_{2}}\right) \cdot \frac{\partial u_{e \mid r_{2}}}{\partial q_{2}},
\end{aligned}
$$


where we apply (see Eqs. (15) and (16)):

$$
\frac{\partial u_{e \mid r_{i}}}{\partial q_{2}}=-\frac{\partial u_{s \mid r_{i}}}{\partial q_{2}} .
$$

Since $\left(p_{e \mid r_{1}}-p_{s \mid r_{1}}\right)<\left(p_{e \mid r_{2}}-p_{s \mid r_{2}}\right)$, for any $(0<) \partial u_{e \mid r_{1}} / \partial q_{2}<\partial u_{e \mid r_{2}} / \partial q_{2}$, we obtain:

$$
\left(p_{e \mid r_{1}}-p_{s \mid r_{1}}\right) \cdot \frac{\partial u_{e \mid r_{1}}}{\partial q_{2}}<\left(p_{e \mid r_{2}}-p_{s \mid r_{2}}\right) \cdot \frac{\partial u_{e \mid r_{2}}}{\partial q_{2}}
$$

which implies $d_{1}-c_{2}^{2}<0$. In a similar way, $d_{3}$ and $c_{2}^{3}$ are respectively given by:

$$
\begin{aligned}
d_{3} & =\frac{\partial S_{r_{3}}\left(q_{3}\right)}{\partial u_{e \mid r_{3}}} \cdot \frac{\partial u_{e \mid r_{3}}}{\partial q_{3}}+\frac{\partial S_{r_{3}}\left(q_{3}\right)}{\partial u_{s \mid r_{3}}} \cdot \frac{\partial u_{s \mid r_{3}}}{\partial q_{3}} \\
& =\left(p_{e \mid r_{3}}-p_{s \mid r_{3}}\right) \cdot \frac{\partial u_{e \mid r_{3}}}{\partial q_{3}}, \\
c_{3}^{2} & =\frac{\partial S_{r_{2}}\left(q_{2}, q_{3}\right)}{\partial u_{e \mid r_{2}}} \cdot \frac{\partial u_{e \mid r_{2}}}{\partial q_{3}}+\frac{\partial S_{r_{2}}\left(q_{2}, q_{3}\right)}{\partial u_{s \mid r_{2}}} \cdot \frac{\partial u_{s \mid r_{2}}}{\partial q_{3}} \\
& =\left(p_{e \mid r_{2}}-p_{s \mid r_{2}}\right) \cdot \frac{\partial u_{e \mid r_{2}}}{\partial q_{3}} .
\end{aligned}
$$

Since $\left(p_{e \mid r_{3}}-p_{s \mid r_{3}}\right)>\left(p_{e \mid r_{2}}-p_{s \mid r_{2}}\right)$, for any $(0<) \partial u_{e \mid r_{2}} / \partial q_{3}<\partial u_{e \mid r_{3}} / \partial q_{3}$, we obtain:

$$
\left(p_{e \mid r_{3}}-p_{s \mid r_{3}}\right) \cdot \frac{\partial u_{e \mid r_{3}}}{\partial q_{3}}>\left(p_{e \mid r_{2}}-p_{s \mid r_{2}}\right) \cdot \frac{\partial u_{e \mid r_{2}}}{\partial q_{3}}
$$

which implies $d_{3}-c_{2}^{3}>0$. As the results, it is clarified that both $A$ and $B$ are negative constants. We have proven that $S\left(\tilde{\mathbf{q}}^{*}\right)$ is strictly a concave function, since $S\left(\widetilde{\mathbf{q}}^{*}\right)$ can be approximated by a strictly concave function of $\Delta q_{2}$ and $\Delta q_{3}$ regardless of feasible $\tilde{\mathbf{q}}^{*}$ at which approximation is made. In addition, since the constraint shown by Eq. (21) for the problem is a convex set, the problem for the local authority has unique solution.

\section{NUMERICAL EXPERIMENTS}

This section shows the present value of the cost to the resident $G(T)$ caused by the landslide. The cost is calculated by applying the Monte Carlo technique by using uniform distribution and the given probabilities associated with the formulation. We assume the simulation term $(T)$ of 50 years and the sampling number $(S)$ of 10,000 which represents the number of residents, and then calculate the mean and variance of the present values of the cost. The probability $p_{f}$ where $f=1$ is assumed as 0.005 . The parameter for the learning process is assumed as $\alpha=0.5$. The costs that relate to the utility functions are assumed as $l_{e}=0.05, l_{m_{0}}=0.00, l_{m_{1}}=10$ and $l_{m_{2}}=20$. The dispersion parameter shown in Eq. (17) is assumed as 1 . The discount rate of 0 is assumed for the simplified understanding of the results from the model. $q_{1}$, and $q_{4}$ which is $q_{\max }$, are assumed as 100 and 300, respectively. The probabilities associated with the model are assumed as: 


$$
p_{* \mid f}(q)=\frac{1}{1+\exp (\mu \cdot(a \cdot(q-\varphi)+b))},
$$

by using the sets of parameters shown in Table 2 . The resulting probabilities where $f=1$, i.e. $p_{q \mid f}$ and $p_{m_{j} \mid f}(j=0,1)$ are shown in Fig. 3 and Fig. 4, respectively.

Table 2. Parameters for probabilities

\begin{tabular}{|c|c|c|c|c|}
\hline$*$ & $a$ & $b$ & $\varphi$ & $\mu$ \\
\hline$q$ & 1.5 & -100 & 110 & 0.05 \\
\hline$m_{0}$ & 1.0 & -150 & 0 & 1 \\
\hline$m_{1}$ & 1.0 & -150 & 70 & 1 \\
\hline
\end{tabular}

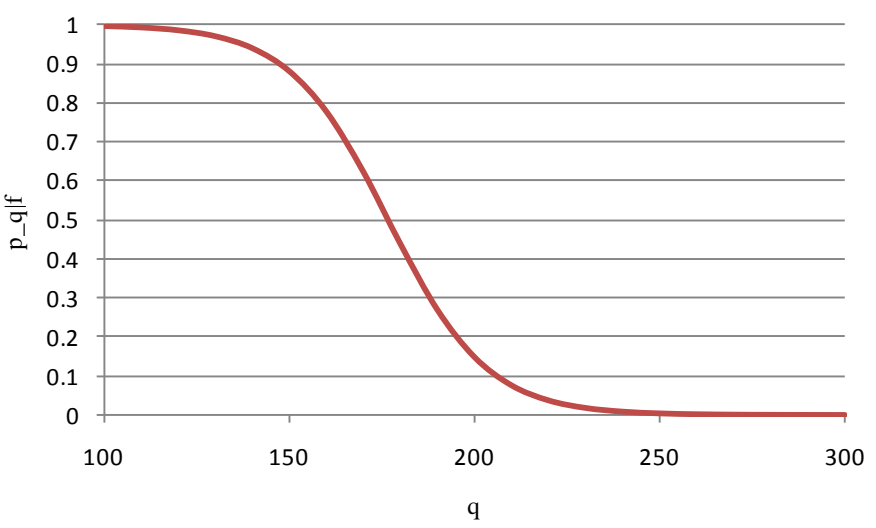

Fig. 3. Rainfall distribution

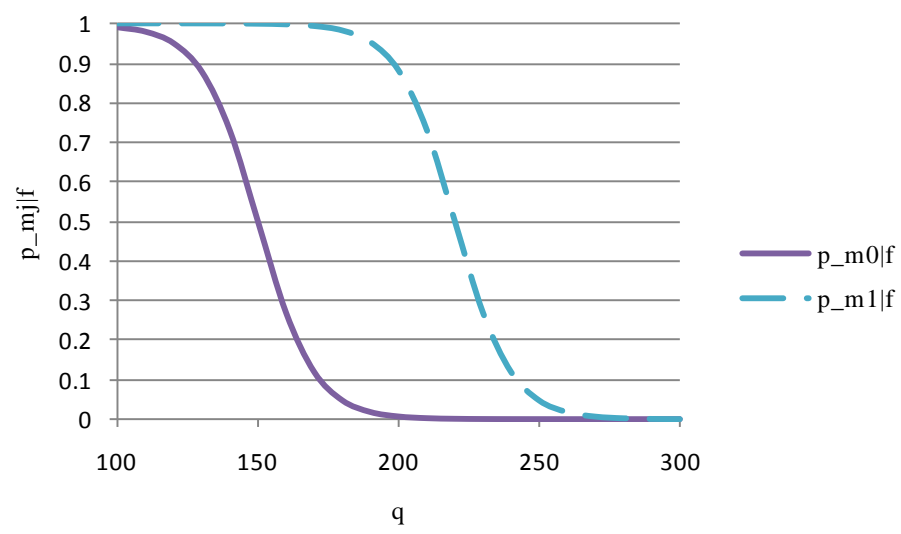

Fig. 4. Landslide probability

The initial evacuation probability will be calculated according to Eq. (17) by using the issuance conditions for the disaster warnings $\mathbf{q}=\left(\begin{array}{ll}q_{2} & q_{3}\end{array}\right)^{T}$. Fig. 5 shows the flowchart of the simulation in which we assume $f=1$. In Fig. 5, the subscript $\_s$ put beside a probability means that the probability is 
drawn from the uniform distribution of $\operatorname{Uni}(0,1)$ as the $s$ th sample.

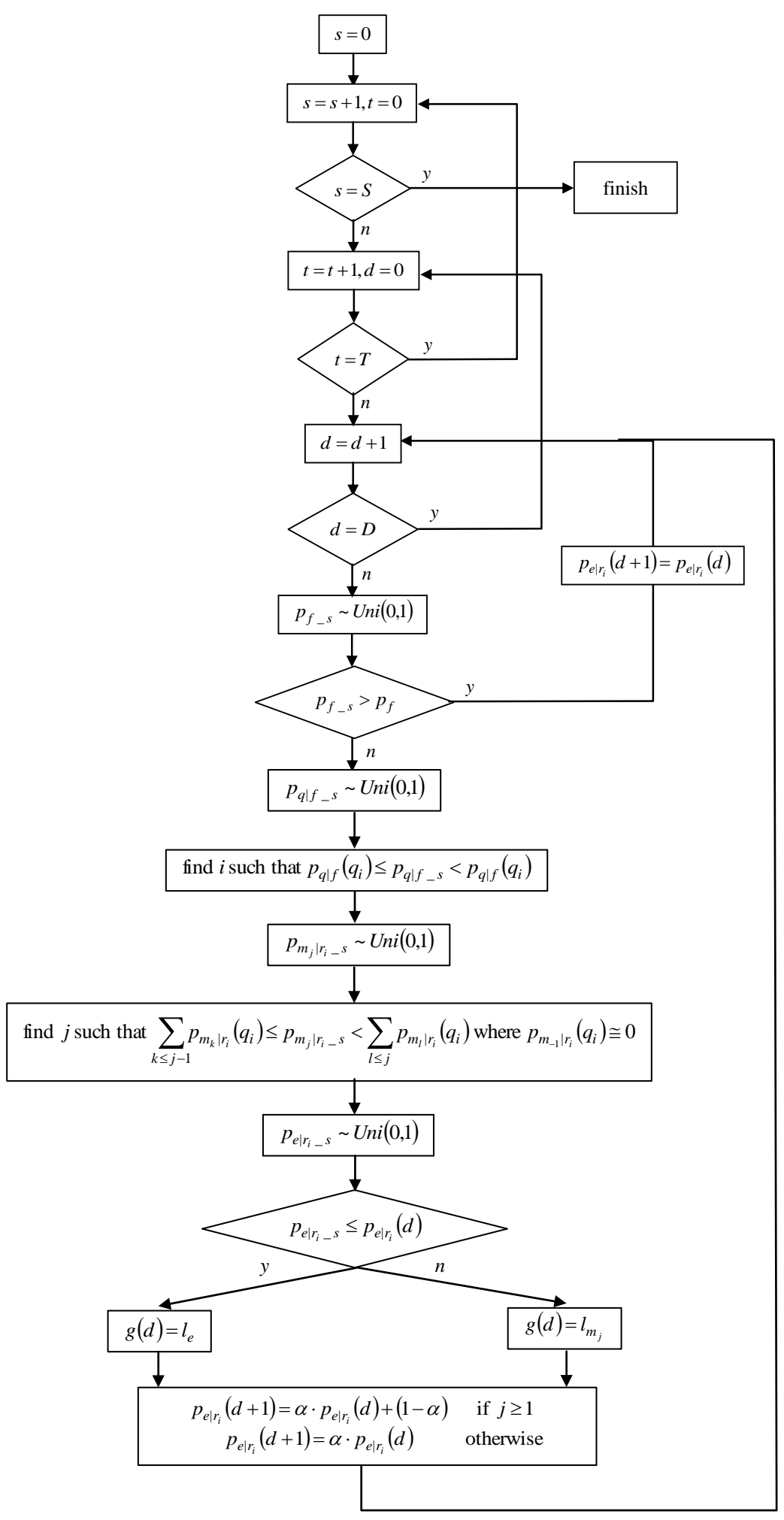

Figure 5. Flowchart of the simulation.

Fig. 6 shows the distribution of the cost $G(T)$ calculated under the issuance conditions of $\mathbf{q}^{1}=\left(q_{1}^{1}=150 \quad q_{2}^{1}=200\right)^{T}$. We call these issuance conditions "issuance conditions 1." Although the 
distribution has two peaks, we still calculate the mean and the variance of the distribution for the purpose of comparing the mean and the variance with those calculated by using different issuance conditions. As a result, the mean of 4.72 and the variance of 12.12 are obtained. The left-hand side of the peaks in Fig. 6 expresses the distribution of cost when the resident does not have to pay for personal suffering from the landslide. The right-hand side of the peak expresses the distribution of the cost when the resident has to pay twice for personal suffering from a landslide with the casualty of $m_{1}$ or once from a landslide with the casualty of $m_{2}$. Fig. 7 shows the distribution of the cost which the resident has to pay calculated under the issuance conditions of $\mathbf{q}^{2}=\left(\begin{array}{lll}q_{1}^{2}=120 & q_{2}^{2}=130\end{array}\right)^{T}$. We will call these issuance conditions "issuance conditions 2." The mean of 6.43 and the variance of 18.03 are obtained with respect to the present value of the cost. Different from Fig. 6, there are four peaks in Fig. 7. Two new peaks appear around the costs of 15 and 35. The former peak expresses the distribution of the cost when the resident pays for personal suffering from a landslide with a casualty of $m_{1}$ once. The other peak, which may be difficult to see, expresses the distribution of the cost when the resident pays for personal suffering more than twice. Since issuance conditions 2 relax issuing $r_{i}$ at $q_{i}^{1}-\delta q_{i}(i=2,3)$ where $\delta q_{2}=30$ and $\delta q_{3}=70$ compared with those defined in issuance conditions 1 , the landslide is not likely to occur before the issuance of disaster warnings under these conditions. However, under issuance conditions 2, the resident is likely to ignore the disaster warnings since the warnings are inaccurate. Therefore, the new peak that appears under issuance conditions 2 is thought to be generated as a result of the resident's disregard of the disaster warnings. The results shown by Figs. 6 and 7 can be thought to point out the fact, which is also the hypothesis of this study, that the issuance conditions for the disaster warnings should be neither too relaxed nor too tight. Under the relaxed issuance conditions, e.g. under issuance conditions 2 , it is clarified that the resident's cost can be huge even though the probability of the landslide occurring may be small.

Fig. 8 shows the distribution of the cost under the optimized issuance conditions. The optimized issuance conditions are estimated as $\mathbf{q}=\left(\begin{array}{lll}137 & 152\end{array}\right)^{T}$, at which the mean and the variance of the cost are calculated as 4.67 and 5.13, respectively. Under the optimized issuance conditions, the variance of the cost decreases greatly, whereas the mean of the cost decreases a little compared with those under the issuance conditions 1. Under the optimized issuance conditions, the resident rarely pays for personal suffering.

Fig. 9 shows the distribution of cost under issuance conditions 1 when the parameter for the learning process is set as $\alpha=0.1$. The mean and the variance of distribution are calculated as 4.70 and 12.10, respectively. Fig. 10 shows the distribution of cost under the same parameter settings as for Fig. 9 but with optimized issuance conditions. The mean and the variance of distribution in this case are calculated as 4.65 and 5.06, respectively. A comparison of the results between Fig. 6 and Fig. 9 shows them to be almost the same, as does a comparison of the results between Fig. 8 and Fig. 10. Thus, it can be concluded 
that the learning effect parameter does not have much influence on the present value of cost within the range of valued studied here.

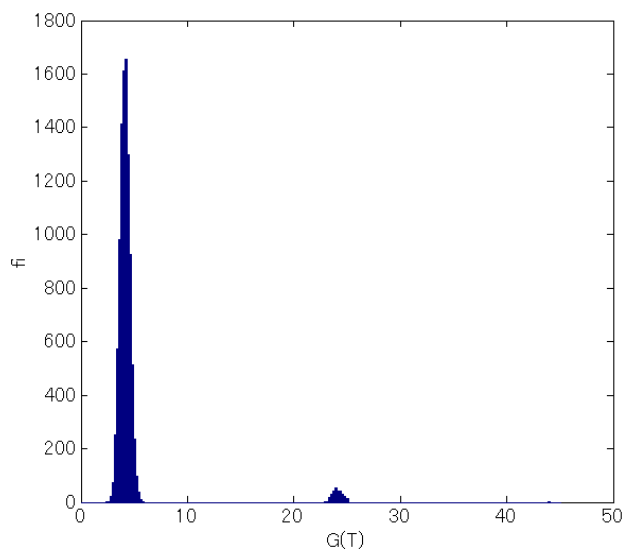

Fig. 6. Distribution of cost under issuance conditions 1

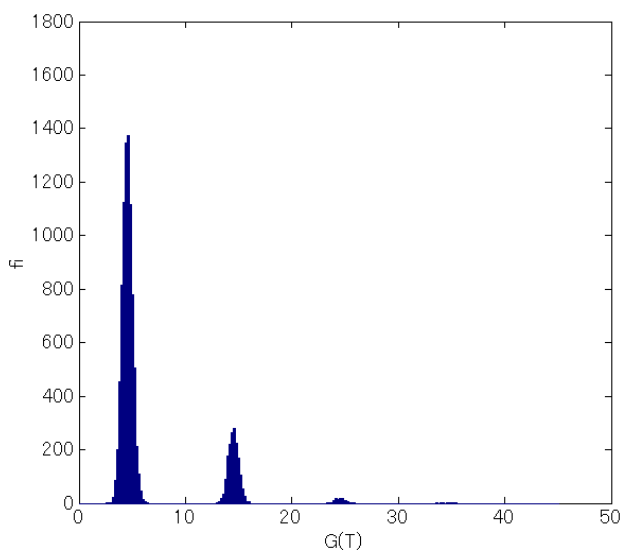

Fig. 7. Distribution of cost under issuance conditions 2

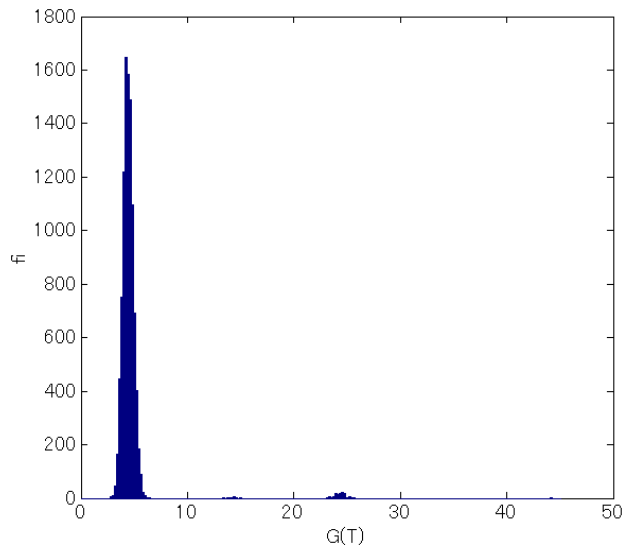

Fig. 8. Distribution of cost under the optimized issuance conditions 


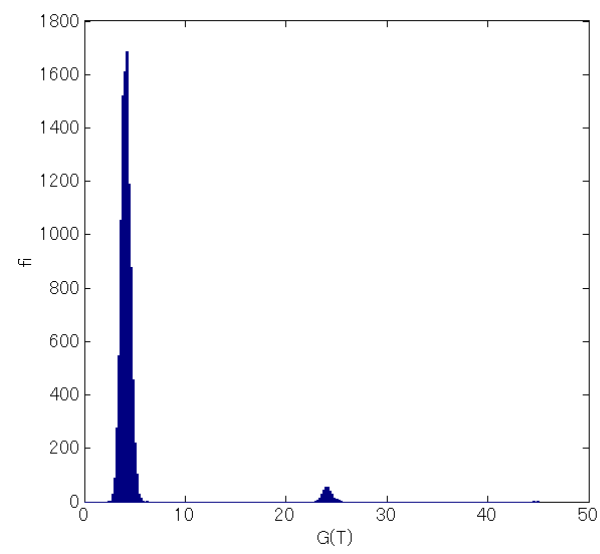

Fig. 9. Distribution of cost under issuance conditions $1(\alpha=0.1)$

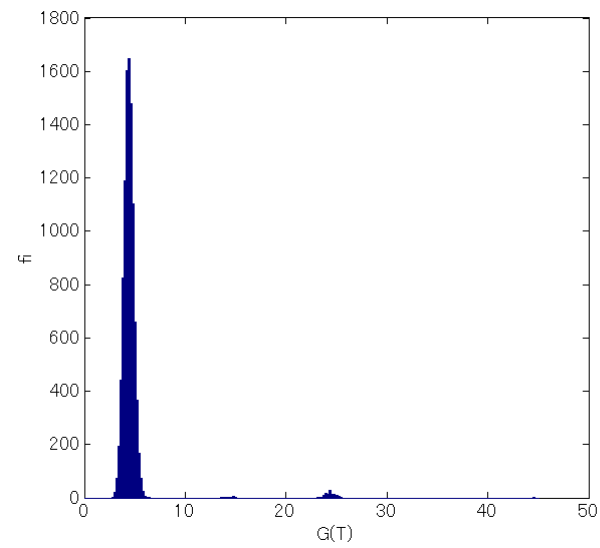

Fig. 10. Distribution of cost under the optimized issuance conditions $(\alpha=0.1)$

\section{CONCLUSIONS}

In this study, a simulation model was proposed that evaluates "cry wolf" syndrome by expressing the behavior of the local authority, which decides the disaster warning issuance conditions, and of the residents, who make a decision to evacuate based on disaster warnings and personal experience. Uncertainty in the decision-making process of the resident as well as the learning process of the resident with respect to the evacuation probability are expressed in the model. The optimal conditions of disaster warning issuance, i.e. those that maximize the resident's expected utility calculated under the condition of disaster warning issuance, are also expressed in the model. In the model, the cost which the resident has to pay reflects the resident's behavior, which is endogenously determined based on the issuance conditions. Disaster warnings with lower accuracy might be ignored by residents. Such effect can be regarded as an essential problem in considering the residents' evacuation behaviors in natural disasters. Numerical experiments which examine the effects of the disaster warning issuance conditions on the resident's cost were then carried out. In the numerical experiments, since both the costs involved in 
evacuation and the probabilities associated with the model have been assumed, the results presented in this study might not be able to reflect the real resident's evacuation behavior. However, the model developed in this study can be used for examining the optimal disaster issuance conditions provided that the parameters associated with the model are correctly estimated and that the learning process introduced to the model is reasonable.

Lindell et al. (2011) recently reported that hurricane evacuations costs averaged US\$200 per household. Since the costs assumed in numerical experiments mean that $l_{m_{1}}$ is 200 times $l_{e}$ and $l_{m_{2}}$ is 400 times $l_{e}$, the casualty costs calculated based on the hurricane evacuations costs are $l_{m_{1}}=$ US\$ 40,000 and $l_{m_{2}}=$ US\$ 80,000 which seem very high. For the purpose of analyzing the resident's realistic evacuation behavior, the costs involved in evacuation must be estimated accurately and be used in the simulation model. In addition to that, both rainfall intensity probabilities and landslide probabilities are also estimated accurately. For the purpose of estimating these two kinds of probability density functions, long-term observation of the relationship between rainfall intensity and landslide occurrence is required. Lindell (2008) generated a tornado diagram to display the results of a sensitivity analysis for the purpose of addressing the uncertainties involved in evacuation behavior. For the purpose of addressing the uncertainties, we have introduced a Monte Carlo simulation technique in this study. Therefore, the cost distributions were obtained. However, different costs setting will bring out different cost distribution. Therefore, setting of reasonable costs and sensitivity analysis of the costs associated with the model are required for examining the optimal disaster issuance conditions. These two tasks need to be carried out in future study.

\section{REFERENCES}

Atwood, L. E., and Major, A. M. (1998). Exploring the 'cry wolf' hypothesis. International Journal of Mass Emergencies \& Disasters, 16 (3), 279-302.

Bretschneider, S., Kimms, A. (2011). Pattern-based evacuation planning for urban areas. European Journal of Operational Research, doi:10.1016/j.ejor.2011.07.015.

Daganzo, C. (1979). Multinomial Probit: The Theory and Its Application to Demand Forecasting. Academic Press Inc., New York.

Dash, N., and Gladwin, H. (2007). Evacuation decision making and behavioral responses: Individual and household. Natural Hazards Review, 8 (3), 69-77.

Kailiponi, P. (2010). Analyzing evacuation decisions using multi-attribute utility theory (MAUT). Procedia Engineering, 3, 163-174.

Lindell, M.K. (2008). EMBLEM2: An empirically based large-scale evacuation time estimate model. Transportation Research A 42, 140-154. 
Lindell, M.K., Kang, J.E. \& Prater, C.S. (2011). The logistics of household evacuation in Hurricane Lili. Natural Hazards. DOI 10.1007/s11069-011-9715-x.

Nakatani, M., Suzuki, D., Sakata N., Nishida, S. (2009a). A study of auditory warning signals for the design guidelines of man-machine interfaces. M.J. Smith and G. Salvendy (Eds.): Human Interface, Part II, HCII 2009, LNCS 5618, 826-834.

Nakatani, M., Suzuki, D., Sakata N., Nishida, S. (2009b). A study of a sense of crisis from auditory warning signals. Proceedings of the World Congress on Engineering and Computer Science 2009, Vol I.

Saadatseresht, M., A. Mansourian, M. Taleai (2009). Evacuation planning using multi-objective evolutionary optimization approach. European Journal of Operational Research, 198: 305-314.

Sorensen, J.H. (2000). Hazard warning systems: Review of 20 years of progress. Natural Hazards Review, $1,119-125$.

Tamura, H., Yamamoto, K., Tomiyama, S., Hatono, I. (2000). Modeling and analysis of decision making problem for mitigating natural disaster risk. European Journal of Operational Research, 122, 461-468.

Wolshon, B., Urbina, E., Wilmot, C., Levitan, M. (2005). Review of Policies and Practices for Hurricane Evacuation. I: Transportation Planning, Preparedness, and Response. Natural Hazards Review, 6 (3), 129-142.

Yi, W., Özdamar, L. (2007). A dynamic logistics coordination model for evacuation and support in disaster response activities. European Journal of Operational Research, 179 (3), pp. 1177-1193. 